\title{
Large Hecke eigenvalues and an Omega result for non-Saito-Kurokawa lifts
}

\author{
Pramath Anamby ${ }^{1}$ (D) Soumya Das ${ }^{2,3} \cdot$ Ritwik Pal $^{2}$ \\ Received: 14 January 2020 / Accepted: 21 August 2020 \\ (c) Springer Science+Business Media, LLC, part of Springer Nature 2020
}

\begin{abstract}
We prove a result on the distribution of Hecke eigenvalues, $\mu_{F}\left(p^{r}\right)$ (for $r=1,2$ or 3 ) of a non-Saito-Kurokawa lift $F$ of degree 2. As a consequence, we obtain an Omega result for the Hecke eigenvalues for such an $F$, which is the best possible in terms of orders of magnitude.
\end{abstract}

Keywords Hecke eigenvalues · Non-Saito-Kurokawa lifts · Omega results

Mathematics Subject Classification Primary 11F46

\section{Introduction}

The study of behavior of Hecke eigenvalues has been an interesting as well as an important theme in the theory of modular forms. For example, the distribution of

\begin{abstract}
S.D. was supported by a Humboldt Fellowship from the Alexander von Humboldt Foundation at Universität Mannheim during the preparation of the paper, and thanks both for the generous support and for providing excellent working conditions. He also thanks IISc, Bangalore, DST (India) and UGC centre for advanced studies for financial support. During the preparation of this work S.D. was supported by a MATRICS Grant MTR/2017/000496 from DST-SERB, India. P.A. and R.P. were supported by IISc Research Associateship during the preparation of this article and thank IISc, Bangalore for the support.
\end{abstract}

$凶$ Pramath Anamby

pramathav@hri.res.in; pramath.anamby@gmail.com

Soumya Das

soumya@iisc.ac.in; sdas@mail.uni-mannheim.de

Ritwik Pal

ritwikpal@iisc.ac.in; ritwik.1729@gmail.com

1 Department of Mathematics, Harish-Chandra Research Institute, Prayagraj (Allahabad) 211019, India

2 Department of Mathematics, Indian Institute Of Science, Bangalore 560012, India

3 Universität Mannheim, Mannheim, Germany 
Hecke eigenvalues and Omega results (i.e., 'sharp' lower bounds on suitable subsequences) have been studied extensively. In the case of an elliptic Hecke eigenform, the equidistribution of the eigenvalues is a consequence of Sato-Tate conjecture, which is known from the deep results in [2]. However, reasonable Omega results can, in many cases, be proved by less sophisticated techniques. For example it is well known that for holomorphic cusp forms on GL(2), such a result follows from the fact that for $r \leq 4$, the symmetric $r$-power $L$-functions have an analytical continuation up to $\operatorname{Re}(s) \geq 1 / 2$ (see [13]).

In the case of our interest, namely holomorphic Siegel modular forms of degree 2, none of the above-mentioned results are known outside of the Maaß space, even though there are some average results [9] (vertical Sato-Tate on average) and [16] (Sato-Tate on average). There are far fewer results however, when one fixes the modular form. Namely, in the case at hand, the distribution of eigenvalues $\lambda_{F}(p)$ ( $p$ prime, $F$ is a non-Saito-Kurokawa lift) can be found in [15] and [4].

In this article we study the distribution of Hecke eigenvalues $\mu_{F}\left(p^{r}\right)$ (for $r=$ $1,2,3, p$ being a prime) of a Siegel Hecke eigenform of degree 2 with full level that is not a Saito-Kurokawa (Maaß) lift. We do this with an aim of proving an Omega result for Hecke eigenvalues of such an $F$. Let us denote by $S_{k}^{2, *}$, the Maaß subspace and by $S_{k}^{2, \perp}$, the subspace of $S_{k}^{2}$ orthogonal to $S_{k}^{2, *}$. Our main result Theorem 1 implies (via Theorem 2) in particular that the Ramanujan-Petersson conjecture for eigenforms in $S_{k}^{2, \perp}$ is optimal, in a sense described below.

First, let us describe the main result of this article. Let $F \in S_{k}^{2, \perp}$ be a Hecke eigenform with eigenvalues $\mu_{F}(n)$; so that if $T(n)$ denotes the $n$-th (similitude) Hecke operator on $S_{k}^{2}$, one has $T(n) F=\mu_{F}(n) F$ for all $n \geq 1$. The Ramanujan-Petersson conjecture (proved by Weissauer [17]) for $F$ implies that (see [7]) for all $n \geq 1$,

$$
\left|\mu_{F}(n)\right| \leq d_{5}(n) n^{k-3 / 2}
$$

where $d_{5}(n)$ denotes the number of ways of writing $n$ as the product of 5 positive integers. We now normalize $\mu_{F}(n)$ by putting

$$
\lambda_{F}(n)=\frac{\mu_{F}(n)}{n^{k-3 / 2}} .
$$

We call $\mu_{F}(n)$ 'large' if $\left|\lambda_{F}(n)\right|>c$ for some $c>1$. Our main theorem then says that there exist a plethora of 'large' eigenvalues if we search in the sequence $\left\{p^{j} \mid p\right.$ prime $\left., j=1,2,3\right\}$. By multiplicativity of the Hecke eigenvalues, this would then produce other 'large' eigenvalues.

Theorem 1 Let $F \in S_{k}^{2, \perp}$ be a Hecke eigenform. Then there exists $c>1$ and $\delta>0$ such that

$$
\liminf _{x \rightarrow \infty} \frac{\#\left\{p \leq x: \max \left\{\left|\lambda_{F}\left(p^{i}\right)\right|: i=1,2,3\right\} \geq c\right\}}{\pi(x)}>\delta,
$$

where $\pi(x)$ denotes the number of primes up to $x$. 
Note that this would mean that for every large $x$, there exists an $i=i_{x} \in\{1,2,3\}$ such that $\#\left\{p \leq x:\left|\lambda\left(p^{i}\right)\right|>c\right\}>\delta \cdot \pi(x)$. This immediately gives us the following corollary.

Corollary 1.1 For at least one $j \in\{1,2,3\}$, the following statement is true: there exist constants $c>1$ and $\delta>0$ such that

$$
\limsup _{x \rightarrow \infty} \frac{\#\left\{p \leq x:\left|\lambda\left(p^{j}\right)\right| \geq c\right\}}{\pi(x)}>\delta .
$$

The main point to note here is that $c>1$ (so that we are dealing with 'large' eigenvalues); analogous assertions when $c<1$ follow already from [4].

The main tools used in the proof of Theorem 1 are the prime number theorems for the spinor and the standard $L$ functions (denoted as $Z(F, s)$ and $Z^{\text {st }}(F, s)$, respectively) attached to $F$ and the Hecke relations among the Hecke eigenvalues. Also crucially used in the proof is the existence of a functorial transfer from GSp(4) to GL(4) from the work of [12], which enables us to use the analytic machinery from GL(4) automorphic representations.

Let us now discuss some applications of Theorem 1 towards Omega results on the sequence of eigenvalues $\left\{\lambda_{F}(n)\right\}$. In particular, we would like to know if (1.1) is the best possible. This means two things: first, the exponent $k-3 / 2$ should be the best possible and second, the order of magnitude of the slowly growing function $d_{5}(n)$ should also be the best possible. Of these, the assertion about the exponent is true and follows from [4]. It should also follow by considering the Rankin-Selberg convolution of $Z(F, s)$ with itself, and arguing with the location of poles (cf. [3, Remark 5.3]). More subtle is the slowly growing function, and we prefer to treat these functions simultaneously.

To set the stage, let us recall some facts about this type of questions in the case of elliptic modular forms and Saito-Kurokawa lifts. In the case of elliptic modular forms, the answer to the question of sharpness of the Ramanujan-Petersson conjecture

$$
\text { (Deligne's bound): }\left|a_{g}(m)\right| \leq d_{2}(n) n^{(k-1) / 2}, \quad n \geq 1
$$

(for a new form $g$ ) is that it is the best possible in terms of the exponent $(k-1) / 2$; so the question boils down to understanding the behavior of the function $a_{g}(m) / m^{(k-1) / 2}$. One knows the following $\Omega$-type results about this function. In [14] Rankin proved, essentially exploiting the prime number theorem for a Hecke eigenform $g \in S_{k}$, that it is not bounded:

$$
\limsup _{m \rightarrow \infty} \frac{a_{g}(m)}{m^{(k-1) / 2}}=\infty .
$$

Even a stronger result is known due to Ram Murty (cf. [13], using the holomorphy of suitable symmetric power $L$-functions):

$$
\frac{a_{g}(m)}{m^{(k-1) / 2}}=\Omega\left(\exp \left(\frac{\alpha \log m}{\log \log m}\right)\right) \quad(\alpha>0) .
$$


It is known that the Saito-Kurokawa lifts of degree 2 fail to satisfy (1.1). Instead they satisfy

$$
\lambda_{F}(n) \ll_{\epsilon} n^{k-1+\epsilon} \text { for any } \epsilon>0 .
$$

An Omega result for such an $F \in S_{k}^{2}$ was obtained by Das (see [6]) and was later improved by Gun et al (see [8]).

Here and in the rest of the paper, for arithmetical functions $f(n), g(n)$ with $g(n)>0$ for all $n \geq 1$, we use the notation

$$
f(n)=\Omega_{ \pm}(g(n)) \quad \text { if and only if } \quad \limsup _{n \rightarrow \infty} \frac{f(n)}{g(n)}>0 \quad\left(\text { resp. } \liminf _{n \rightarrow \infty} \frac{f(n)}{g(n)}<0\right) .
$$

In more simple terms, this just means that $|f(n)| / g(n)$ is bounded away from zero along a subsequence of the set of natural numbers $\mathbb{N}$. Moreover we write $f(n)=$ $\Omega(g(n))$ if $|f(n)|=\Omega_{+}(g(n))$.

Using the Corollary 1.1 of Theorem 1, we can deduce easily the following Omega result.

Theorem 2 Let $F$ be as in Theorem 1. Then there exists a constant $c>0$ such that

$$
\lambda_{F}(n)=\Omega_{ \pm}\left(\exp \left(\frac{c \log n}{\log \log n}\right)\right)
$$

Actually the above Omega result is realized over a certain subset of fourth power-free integers. It is easy to check that on this subset $\log d_{5}(n)$ and the function $\log n / \log \log n$ are the same asymptotically up to the constant $c$. For example, if $p$ is a prime, then $d_{5}(p)=5$. Therefore putting $a_{N}=\prod_{p \leq N} p, \log d_{5}\left(a_{N}\right)=\log 5 \cdot \log (\pi(N)) \sim$ $A \cdot \log a_{N} / \log \log a_{N}$. So our result can also be presented as $\lambda_{F}(n)=\Omega_{ \pm}\left(d_{5}(n)^{\omega}\right)$ for some $\omega>0$. At any rate this not only proves the optimality of the exponent in (1.1), but that the slowly growing function is also the same up to a suitable exponent.

It is also interesting to ask for Omega results in the context of Fourier coefficients; this has recently been addressed in [5]. It is not immediately clear how the results of this article influence those of [5] and vice versa.

\section{Notation and preliminaries}

First we recall some basic facts about Siegel cusp forms of degree 2 and the classical $L$-functions attached to them. Let $F \in S_{k}^{2}$ be an eigenform for all Hecke operators $T(n)$ which is not a Saito-Kurokawa lift. Let $\left\{\lambda_{F}(n)\right\}$ (normalized as in (1.2)) be the normalized eigenvalues of $F$. We refer the reader to [1] for more details. 


\section{Some $L$-functions attached to $F$}

The degree 4 spinor zeta function attached to $F$ is given by

$$
Z(F, s)=\prod_{p} Z_{p}\left(F, p^{-s}\right),
$$

where the $p$-th Euler factor $Z_{p}(F, \cdot)$ of $Z(F, \cdot)$ is given by

$$
\begin{aligned}
Z_{p}(F, t)^{-1} & =\left(1-\alpha_{0, p} t\right)\left(1-\alpha_{0, p} \alpha_{1, p} t\right)\left(1-\alpha_{0, p} \alpha_{2, p} t\right)\left(1-\alpha_{0, p} \alpha_{1, p} \alpha_{2, p} t\right) \\
& =1-\lambda_{F}(p) t+\left(\lambda_{F}(p)^{2}-\lambda_{F}\left(p^{2}\right)-p^{-1}\right) t^{2}-\lambda_{F}(p) t^{3}+t^{4} .
\end{aligned}
$$

The degree 5 standard $L$-function attached to $F$ is given by

$$
Z^{\mathrm{st}}(F, s)=\prod_{p} Z_{p}^{\mathrm{st}}\left(F, p^{-s}\right),
$$

where

$$
Z_{p}^{\mathrm{st}}(F, t)^{-1}=(1-t)\left(1-\alpha_{1, p} t\right)\left(1-\alpha_{2, p} t\right)\left(1-\alpha_{1, p}^{-1} t\right)\left(1-\alpha_{2, p}^{-1} t\right) .
$$

Here $\alpha_{0, p}, \alpha_{1, p}, \alpha_{2, p}$ denote the Satake $p$-parameters attached to $F$ and satisfy

$$
\alpha_{0, p}^{2} \alpha_{1, p} \alpha_{2, p}=1
$$

By virtue of the Ramanujan-Petersson conjecture proved by Weissauer (see [17]), we have

$$
\left|\alpha_{0, p}\right|=\left|\alpha_{1, p}\right|=\left|\alpha_{2, p}\right|=1
$$

for all primes $p$. Moreover the Hecke eigenvalues are related to the spinor zeta function by

$$
\sum_{n \geq 1} \lambda_{F}(n) n^{-s}=\frac{Z(F, s)}{\zeta(2 s+1)} .
$$

Let the Dirichlet series of $Z^{\text {st }}(F, s)$ be denoted as

$$
Z^{\mathrm{st}}(F, s)=\sum_{n \geq 1} \frac{b(n)}{n^{s}} .
$$

Then by expanding (2.2) we have

$$
b(p)=1+\alpha_{1, p}+\alpha_{2, p}+\alpha_{1, p}^{-1}+\alpha_{2, p}^{-1} .
$$

From [12] we know that there exist cuspidal automorphic representations $\Pi_{4}$ of $\mathrm{GL}_{4}(\mathbb{A})$ and $\Pi_{5}$ of $\mathrm{GL}_{5}(\mathbb{A})$ such that

$$
Z(F, s)=L\left(\Pi_{4}, s\right) \quad \text { and } \quad Z^{\text {st }}(F, s)=L\left(\Pi_{5}, s\right) .
$$


Then using the prime number theorem (PNT) for Rankin-Selberg $L$ functions $L\left(\Pi_{4} \times\right.$ $\left.\Pi_{4}, s\right)$ and $L\left(\Pi_{5} \times \Pi_{5}, s\right)$ (see [11]), we obtain

Lemma 2.1 For all large $X$

(1) $\sum_{p \leq X} \lambda_{F}(p)^{2} \log p=X+O\left(X \exp \left(-\kappa_{1} \sqrt{\log X}\right)\right)$.

(2) $\sum_{p \leq X} b(p)^{2} \log p=X+O\left(X \exp \left(-\kappa_{2} \sqrt{\log X}\right)\right)$.

Here $\kappa_{1}, \kappa_{2}>0$.

\section{Hecke relations}

The eigenvalues $\lambda_{F}\left(p^{n}\right)$ of $F$ satisfy the following recursive relation (from [1, Theorem 1.3.2]).

$$
\begin{gathered}
\lambda_{F}\left(p^{n}\right)=\lambda_{F}(p)\left(\lambda_{F}\left(p^{n-1}\right)+\lambda_{F}\left(p^{n-3}\right)\right)-\lambda_{F}\left(p^{n-2}\right)\left(\lambda_{F}(p)^{2}-\lambda_{F}\left(p^{2}\right)-\frac{1}{p}\right) \\
-\lambda_{F}\left(p^{n-4}\right) .
\end{gathered}
$$

We also need the relation between the eigenvalues $\lambda_{F}(p), \lambda_{F}\left(p^{2}\right)$ and the Dirichlet coefficients $b(p)$ of the standard $L$-function $Z^{\text {st }}(F, s)$. Let $\beta_{1, p}=\alpha_{0, p}, \beta_{2, p}=$ $\alpha_{0, p} \alpha_{1, p}, \beta_{3, p}=\alpha_{0, p} \alpha_{2, p}$ and $\beta_{4, p}=\alpha_{0, p} \alpha_{1, p} \alpha_{2, p}$. The $p$-th Euler factor of $Z(F, s)$ can be written in terms of $\beta_{i, p}$ s as follows:

$$
Z_{p}(F, t)^{-1}=\prod_{1 \leq i \leq 4}\left(1-\beta_{i, p} t\right) .
$$

By expanding the product and using (2.1) we get the following identities. Namely

$$
\lambda_{F}(p)=\sum_{1 \leq i \leq 4} \beta_{i, p}
$$

and

$$
\lambda_{F}(p)^{2}-\lambda_{F}\left(p^{2}\right)-p^{-1}=\sum_{1 \leq i<j \leq 4} \beta_{i, p} \beta_{j, p} .
$$

Combining these identities we get

$$
\lambda\left(p^{2}\right)=\sum_{1 \leq i \leq j \leq 4} \beta_{i, p} \beta_{j, p}-\frac{1}{p}
$$

From (2.7), (2.9) and (2.5) one obtains the following estimates:

$$
\left|\lambda_{F}(p)\right| \leq 4, \quad\left|\lambda_{F}\left(p^{2}\right)\right| \leq 10+1 / p, \quad|b(p)| \leq 5 .
$$

We also need the relation between the eigenvalues $\lambda(p), \lambda\left(p^{2}\right)$ and the Dirichlet coefficient $b(p)$ of the standard $L$-function. We get the following relation by using the 
identities (2.3), (2.5), (2.7) and (2.8):

$$
\lambda_{F}(p)^{2}-\lambda_{F}\left(p^{2}\right)=b(p)+1+\frac{1}{p} .
$$

For $a<b$ and $i=1,2$ or 3, we consider the following subsets of $\mathscr{P}$, the set of prime numbers.

$$
V_{i}(a, b ; x):=\left\{p \leq x: a \leq\left|\lambda\left(p^{i}\right)\right|<b\right\}
$$

and we denote the set $\left\{p \leq x:\left|\lambda\left(p^{i}\right)\right| \geq a\right\}$ by $V_{i}(a, \bullet ; x)$. Let us put

$$
\eta_{1}=10^{-10} \text { and } \eta_{2}=1 / 10
$$

\section{Proof of Theorem 1}

In this section we collect various implications arising from the asymptotic formulas of the PNT for $Z(F, s)$ and $Z^{\text {st }}(F, s)$ (cf. Lemma 2.1) in combination with the Hecke relations (2.11) and the bounds on the eigenvalues (2.10). The results are in the form of lower bounds on the sets $V_{j}(a, b ; x)$ under suitable hypotheses.

Note that using the partial summation one can deduce (from Lemma 2.1) that

$$
\sum_{p \leq x} \lambda_{F}(p)^{2}=\frac{x}{\log x}+o\left(\frac{x}{\log x}\right)
$$

and similarly

$$
\sum_{p \leq x} b^{2}(p)=\frac{x}{\log x}+o\left(\frac{x}{\log x}\right) .
$$

\subsection{Choice of $X_{0}$}

We choose a large $X_{0}$ such that the following hold (note here that $X_{0}$ may be dependent on the weight $k$ ).

(1) Let $M(x)$ and $E_{i}(x)(i=1,2)$ denote the main and error terms in (3.1) and (3.2), respectively. Then, for $x>X_{0}, E_{i}(x) \leq 10^{-6} \cdot M(x)$ for $i=1,2$.

(2) For $x \geq X_{0}, \pi\left(10^{4}\right) \leq 10^{-6} \cdot \pi(x)$.

(3) $\frac{999}{1000} \cdot \bar{\pi}(x) \leq \frac{x}{\log x}$ for all $x>X_{0}$.

With this choice of $X_{0}$, we have the following results.

Proposition 3.1 For any $x \geq X_{0}$, one of the following is true.

(i) For some $\delta_{1} \geq 10^{-5},\left|V_{1}\left(1+\eta_{1}, \bullet ; x\right)\right| \geq \delta_{1} \cdot \pi(x)$.

(ii) For some $\delta_{2} \geq 98 / 100$, $\left|V_{1}\left(1-\eta_{2}, 1+\eta_{1} ; x\right)\right| \geq \delta_{2} \cdot \pi(x)$.

Proof Let $x_{0} \geq X_{0}$ such that (i) and (ii) does not hold. That is suppose $\mid V_{1}(1+$ $\left.\eta_{1}, \bullet ; x_{0}\right) \mid<10^{-5} \cdot \pi\left(x_{0}\right)$ and $\left|V_{1}\left(1-\eta_{2}, 1+\eta_{1} ; x_{0}\right)\right|<98 / 100 \cdot \pi\left(x_{0}\right)$. 
Now we decompose the sum on the LHS of (3.1) into disjoint parts and bound them as follows:

$$
\begin{aligned}
\sum_{p \leq x_{0}} \lambda_{F}(p)^{2}= & \sum_{p \in V_{1}\left(0,1-\eta_{2} ; x_{0}\right)} \lambda_{F}(p)^{2}+\sum_{p \in V_{1}\left(1-\eta_{2}, 1+\eta_{1} ; x_{0}\right)} \lambda_{F}(p)^{2} \\
& +\sum_{p \in V_{1}\left(1+\eta_{1}, \bullet ; x_{0}\right)} \lambda_{F}(p) \\
< & \left(1-\eta_{2}\right)^{2}\left|V_{1}\left(0,1-\eta_{2} ; x_{0}\right)\right|+\left(1+\eta_{1}\right)^{2}\left|V_{1}\left(1-\eta_{2}, 1+\eta_{1} ; x_{0}\right)\right| \\
& +16\left|V_{1}\left(1+\eta_{1}, \bullet ; x_{0}\right)\right| .
\end{aligned}
$$

For simplicity, let us put

$$
A:=\left|V_{1}\left(0,1-\eta_{2} ; x_{0}\right)\right|, B:=\left|V_{1}\left(1-\eta_{2}, 1+\eta_{1} ; x_{0}\right)\right|, C:=\left|V_{1}\left(1+\eta_{1}, \bullet ; x_{0}\right)\right|,
$$

so that $A+B+C=\pi\left(x_{0}\right)$. Then

$$
\begin{aligned}
\sum_{p \leq x_{0}} \lambda_{F}(p)^{2}< & \left(1-\eta_{2}\right)^{2} \pi\left(x_{0}\right)+B\left(\left(1+\eta_{1}\right)^{2}-\left(1-\eta_{2}\right)^{2}\right)+C\left(16-\left(1-\eta_{2}\right)^{2}\right) \\
< & \left(\left(1-\eta_{2}\right)^{2}+\frac{98}{100}\left(\left(1+\eta_{1}\right)^{2}-\left(1-\eta_{2}\right)^{2}\right)\right. \\
& \left.+10^{-5}\left(16-\left(1-\eta_{2}\right)^{2}\right)\right) \pi\left(x_{0}\right) \\
& <\frac{998}{1000} \cdot \pi\left(x_{0}\right)
\end{aligned}
$$

after a short calculation. Thus, for any $x$ such that the conditions (i) and (ii) both fail, the RHS is bounded by $998 / 1000 \cdot \pi(x)$. This is clearly a contradiction in view of conditions (1) and (3) in subsection (3.1) on choice of $X_{0}$.

If condition (i) of Proposition 3.1 is true for all $x \geq X_{0}$, then the proof of Theorem 1 is done. But, if for some $x_{0} \geq X_{0}$ only condition (ii) of Proposition 3.1 is true, then we need to look at the sets $V_{2}\left(a, b ; x_{0}\right)$ and $V_{3}\left(a, b ; x_{0}\right)$. To do this we look at the distribution of coefficients $b(p)$ of the standard $L$-function $Z^{\text {st }}(F, s)$.

Proposition 3.2 Let $x_{0} \geq X_{0}$ be such that the condition ( $i$ ) of Proposition 3.1 does not hold. Additionally suppose that $\#\left\{p \leq x_{0}:|b(p)|>2.1\right\}>10^{-3} \cdot \pi\left(x_{0}\right)$. Then

$$
\left|V_{2}\left(1.09, \bullet ; x_{0}\right)\right|>9 \times 10^{-4} \cdot \pi\left(x_{0}\right)
$$

Proof From (2.11) we have

$$
\left|\lambda_{F}\left(p^{2}\right)\right| \geq|b(p)|-\left|1-\lambda_{F}(p)^{2}+p^{-1}\right|
$$


Now for $p \notin V_{1}\left(1+\eta_{1}, \bullet ; x\right)$, note that

$$
\left|1-\lambda_{F}(p)^{2}+1 / p\right| \leq \begin{cases}1+1 / p & \text { if }\left|\lambda_{F}(p)\right| \leq 1 \\ \alpha+1 / p & \text { if } 1<\left|\lambda_{F}(p)\right| \leq 1+\eta_{1}\end{cases}
$$

In the second inequality above we have put $\lambda_{F}(p)^{2}=1+\alpha$ and an easy calculation shows that $0<\alpha<10^{-9}$. Thus it follows from (3.4) that

$$
\left|\lambda_{F}\left(p^{2}\right)\right|>|b(p)|-1-1 / p .
$$

Let us put $A(x):=\{p \leq x:|b(p)|>2.1\}$. Moreover, if $p \in A(x)$ (and $p>10^{4}$ ) we have

$$
\left|\lambda_{F}\left(p^{2}\right)\right|>2.1-1-p^{-1}>1.09 .
$$

These observations suffice to finish the proof as follows. From our two hypotheses in the statement of Proposition 3.2 it follows that

$$
\left|A\left(x_{0}\right)\right|>10^{-3} \pi\left(x_{0}\right) ; \quad\left|V_{1}\left(1+\eta_{1}, \bullet ; x_{0}\right)\right|<10^{-5} \pi\left(x_{0}\right) .
$$

From the above calculations and (3.8) we then conclude (putting $B^{c}=$ 'complement' of $B$ )

$$
A\left(x_{0}\right) \cap V_{1}\left(1+\eta_{1}, \bullet ; x_{0}\right)^{c} \backslash \mathscr{P}\left(10^{4}\right) \subset V_{2}\left(1.09, \bullet ; x_{0}\right),
$$

where $\mathscr{P}\left(10^{4}\right)$ is the set of primes $\leq 10^{4}$. By our choice of $X_{0}$, we have $\pi\left(10^{4}\right) \leq$ $\pi\left(x_{0}\right) / 10^{6}$. Therefore

$$
\begin{aligned}
\left|V_{2}\left(1.09, \bullet ; x_{0}\right)\right| & \geq\left|A\left(x_{0}\right)\right|-\left|V_{1}\left(1+\eta_{1}, \bullet ; x_{0}\right)\right|-\pi\left(10^{4}\right) \\
& \geq\left(10^{-3}-10^{-5}-10^{-6}\right) \pi\left(x_{0}\right)
\end{aligned}
$$

which immediately gives the lemma.

Now we prove a result regarding the coefficients $b(p)$ of $Z^{\text {st }}(F, s)$.

Proposition 3.3 Let $x_{0} \geq X_{0}$ be such that $\#\left\{p \leq x_{0}:|b(p)|>2.1\right\} \leq 10^{-3} \cdot \pi\left(x_{0}\right)$. Then $\#\left\{p \leq x_{0}: 6 / 7 \leq|b(p)| \leq 2.1\right\}>\frac{1}{16} \cdot \pi\left(x_{0}\right)$.

Proof We argue in the same way as in Proportion 3.1. First we decompose the LHS of (3.2) into disjoint sums as follows:

$$
\sum_{p \leq x_{0}} b^{2}(p)=\sum_{\substack{p \leq x_{0} \\ 0 \leq|b(p)|<6 / 7}} b^{2}(p)+\sum_{\substack{p \leq x_{0} \\ 6 / 7 \leq|b(p)| \leq 2.1}} b^{2}(p)+\sum_{\substack{p \leq x_{0} \\ 2.1<|b(p)| \leq 5.1}} b^{2}(p)
$$

As in the proof of Proposition 3.1, let $A, B$ and $C$ denote the cardinality of the sets in the first, second and third terms of the RHS, respectively. Thus we have $A+B+C=\pi\left(x_{0}\right)$ 
and we get

$$
\begin{aligned}
\sum_{p \leq x_{0}} b^{2}(p) & \leq \frac{36}{49}\left(\pi\left(x_{0}\right)-B-C\right)+4.41 \cdot B+25 \cdot C \\
& =\frac{36}{49} \cdot \pi\left(x_{0}\right)+\left(4.41-\frac{36}{49}\right) \cdot B+\left(25-\frac{36}{49}\right) \cdot C .
\end{aligned}
$$

From our assumption we have, $C \leq 10^{-3} \cdot \pi\left(x_{0}\right)$. Now, if the conclusion of the proposition is not true, then $B \leq \frac{1}{16} \cdot \pi\left(x_{0}\right)$ and we have

$$
\begin{aligned}
\sum_{p \leq x_{0}} b^{2}(p) & \leq\left(\frac{36}{49}+3.68 \cdot \frac{1}{16}+24.27 \cdot \frac{1}{10^{3}}\right) \cdot \pi\left(x_{0}\right) \\
& <\frac{989}{1000} \cdot \pi\left(x_{0}\right) .
\end{aligned}
$$

A clear contradiction to (3.2) by our choice of $X_{0}$.

Proposition 3.4 Let $x_{0} \geq X_{0}$ be such that the condition (1) of Proposition 3.1 does not hold. Additionally suppose that $\#\left\{p \leq x_{0}:|b(p)|>2.1\right\} \leq 10^{-3} \cdot \pi\left(x_{0}\right)$. Then

$$
\left|V_{3}\left(1.02, \bullet ; x_{0}\right)\right|>\frac{1}{25} \cdot \pi\left(x_{0}\right) .
$$

Proof We again make use of the following inequality from (2.11).

$$
\left|\lambda_{F}\left(p^{2}\right)\right| \geq|b(p)|-\left|1-\lambda_{F}(p)^{2}+p^{-1}\right| .
$$

For $p \in V_{1}\left(1-\eta_{2}, 1+\eta_{1} ; x\right)$, since $\eta_{2}=\frac{1}{10}$, we have

$$
\left|1-\lambda_{F}(p)^{2}+1 / p\right| \leq \begin{cases}19 / 100+1 / p & \text { if }\left(1-\eta_{2}\right) \leq\left|\lambda_{F}(p)\right| \leq 1 \\ \alpha+1 / p & \text { if } 1<\left|\lambda_{F}(p)\right|<1+\eta_{1}\end{cases}
$$

where $0<\alpha<10^{-9}$. Thus for $p \in V_{1}\left(1-\eta_{2}, 1+\eta_{1} ; x\right) \cap\{p \leq x:|b(p)| \geq 6 / 7\}$ we have

$$
\left|\lambda_{F}\left(p^{2}\right)\right| \geq \frac{6}{7}-\frac{19}{100}-\frac{1}{p} .
$$

Again choosing $p$ large enough $\left(p>10^{4}\right)$ we get that $\left|\lambda_{F}\left(p^{2}\right)\right| \geq 0.667>2 / 3$ and we have

$$
V_{1}\left(1-\eta_{2}, 1+\eta_{1} ; x\right) \cap\{p \leq x:|b(p)| \geq 6 / 7\} \backslash \mathscr{P}\left(10^{4}\right) \subseteq V_{2}(2 / 3, \bullet ; x)
$$

Now from the Hecke relations (see (2.6)) we have

$$
\lambda_{F}\left(p^{3}\right)=\lambda_{F}(p)\left(2 \lambda_{F}\left(p^{2}\right)-\lambda_{F}(p)^{2}+1+\frac{1}{p}\right)
$$


and if $p \in V_{2}\left(2 / 3, \bullet ; x_{0}\right) \cap V_{1}\left(1-\eta_{2}, 1+\eta_{1} ; x_{0}\right)$, we have

$$
\begin{aligned}
\left|\lambda_{F}\left(p^{3}\right)\right| & \geq\left(1-\eta_{2}\right)|| 2 \lambda_{F}\left(p^{2}\right)|-| \lambda_{F}(p)^{2}-1-\frac{1}{p}|| \\
& >\frac{9}{10}\left(\frac{4}{3}-\frac{19}{100}-\frac{1}{p}\right) \\
& >1.02 .
\end{aligned}
$$

Combining this with (3.13), gives us the following inclusions:

$$
\begin{aligned}
V_{3}\left(1.02, \bullet ; x_{0}\right) & \supseteq V_{2}\left(2 / 3, \bullet ; x_{0}\right) \cap V_{1}\left(1-\eta_{2}, 1+\eta_{1} ; x_{0}\right) \\
& \supseteq V_{1}\left(1-\eta_{2}, 1+\eta_{1} ; x_{0}\right) \cap\left\{p \leq x_{0}:|b(p)| \geq 6 / 7\right\} \backslash \mathscr{P}\left(10^{4}\right) .
\end{aligned}
$$

Now since condition (1) of Proposition 3.1 does not hold for $x_{0}, V_{1}\left(1-\eta_{2}, 1+\eta_{1} ; x_{0}\right) \geq$ $\frac{98}{100} \cdot \pi\left(x_{0}\right)$ and from Proposition 3.3, we have $\#\left\{p \leq x_{0}:|b(p)| \geq 6 / 7\right\}>\frac{1}{16} \cdot \pi\left(x_{0}\right)$. Thus

$$
\left|V_{3}\left(1.02, \bullet ; x_{0}\right)\right| \geq\left(\frac{98}{100}+\frac{1}{16}-1-\frac{1}{10^{6}}\right) \cdot \pi\left(x_{0}\right)>\frac{1}{25} \cdot \pi\left(x_{0}\right) .
$$

Proof of Theorem 1 Fix an $x \geq X_{0}$. Now choose $c=1+\eta_{1}$ (which is the smallest among $1+\eta_{1}, 1.09$ and 1.02) and $\delta=10^{-5}$ (which is the smallest among $10^{-5}$, $9 \times 10^{-4}$ and $\left.1 / 25\right)$. Note here that both $c$ and $\delta$ are independent of $x$.

From Propositions 3.1,3.2 and 3.4, for each large enough $x$, we get an $l_{x} \in\{1,2,3\}$ such that $V_{l_{x}}(c, \bullet ; x)>\delta \cdot \pi(x)$. Also note that for any $p \in V_{l_{x}}(c, \bullet ; x)$,

$$
\max \left\{\left|\lambda_{F}\left(p^{i}\right)\right|: i=1,2,3\right\} \geq\left|\lambda_{F}\left(p^{l_{x}}\right)\right| \geq c .
$$

Thus for any $x \geq X_{0}$, there exists an $l_{x} \in\{1,2,3\}$ such that

$$
\left\{p \leq x: \max \left\{\left|\lambda_{F}\left(p^{i}\right)\right|: i=1,2,3\right\} \geq c\right\} \supseteq V_{l_{x}}(c, \bullet ; x) .
$$

This completes the proof of Theorem 1 since both $c$ and $\delta$ are independent of $x$.

Remark 3.5 Note here that the numerical values used in this section are not optimal. This is because it does not improve the Omega result that we are after.

\section{Proof of Theorem 2}

By Corollary 1.1 of Theorem 1, there exist constants $C>1, \delta>0$ and an integer $1 \leq r \leq 3$ (depending on $N$ ) such that

$$
\#\left\{p \leq N:\left|\lambda\left(p^{r}\right)\right| \geq C\right\}>\delta \cdot \pi(N)
$$


for infinitely many integers $N$. Fix the integer $r$ from (4.1) and denote the set on the LHS by $B_{N}$. We now use standard techniques (see [13] for similar arguments) to prove the $\Omega_{ \pm}$result. Let $B_{N}^{+}=\left\{p \leq N: \lambda\left(p^{r}\right) \geq C\right\} \subset B_{N}$ and $B_{N}^{-}=\left\{p \leq N: \lambda\left(p^{r}\right) \leq\right.$ $-C\} \subset B_{N}$. Since $B_{N}>\delta \cdot \pi(N)$, either $B_{N}^{+}>\delta_{1} \cdot \pi(N)$ for some $\delta_{1}>0$ or $B_{N}^{-}>\delta_{2} \cdot \pi(N)$ for some $\delta_{2}>0$.

If $B_{N}^{+}>\delta_{1} \cdot \pi(N)$, choose an integer $n$ as follows:

$$
n=\prod_{p \in B_{N}^{+}} p^{r}
$$

Note here that $r$ varies with $N$ and thus $n$. But this is not a cause of concern since $r \leq 3$.

With this choice of $n$, we have

$$
\lambda_{F}(n)=\prod_{p \in B_{N}^{+}} \lambda_{F}\left(p^{r}\right)
$$

Thus

$$
\lambda_{F}(n) \geq C^{\left|B_{N}^{+}\right|}>C^{\delta_{1} \cdot \pi(N)} \geq C^{\delta_{1} c_{1} \cdot \frac{N}{\log N}}=\exp \left(c_{0} \frac{N}{\log N}\right),
$$

where we choose constants $c_{1}$ and $c_{2}$ such that $c_{1} \frac{N}{\log N} \leq \pi(N) \leq c_{2} \frac{N}{\log N}$ for all large $N$. Now from (4.2) we have

$$
\log n=r \sum_{p \in B_{N}^{+}} \log p \leq r \sum_{p \leq N} \log p \sim r N
$$

Also note that

$$
\log n \geq r \log 2 \cdot\left|B_{N}^{+}\right| \gg \frac{N}{\log N},
$$

from which we get, $\log N \ll \log \log n$. Hence for some constant $c$,

$$
\lambda_{F}(n) \gg \exp \left(\frac{c \log n}{\log \log n}\right)
$$

If $B_{N}^{-}>\delta_{2} \cdot \pi(N)$, we take $n$ to be product of even number of primes in $B_{N}^{-}$and proceed as above.

Now to prove the $\Omega_{-}$result consider the following. If $B_{N}^{+}>\delta_{1} \cdot \pi(N)$, then we proceed as follows. We know that there exists a $n_{0} \in \mathbf{Z}$ such that $\lambda_{F}\left(n_{0}\right)<0$ (see [10]). Now let

$$
n=n_{0} \prod_{\substack{p \in B_{N}^{+} \\\left(p, n_{0}\right)=1}} p^{r}
$$


Thus $\lambda_{F}(n)=\lambda_{F}\left(n_{0}\right) \prod_{\substack{p \in B_{N}^{+} \\\left(p, n_{0}\right)=1}} \lambda_{F}\left(p^{r}\right)$. Now proceeding as above we get

$$
-\lambda_{F}(n) \gg \exp \left(\frac{c \log n}{\log \log n}\right) .
$$

If $B_{N}^{-}>\delta_{2} \cdot \pi(N)$, we take $n$ to be product of odd number of primes in $B_{N}^{-}$and proceed as above. This completes the proof of Theorem 2.

\section{References}

1. Andrianov, A.N.: Euler products corresponding to Siegel modular forms of genus 2. Russ. Math. Surv. 29(3), 45 (1974)

2. Barnet-Lamb, T., et al.: A family of Calabi-Yau varieties and potential automorphy II. Publ. Res. Inst. Math. Sci. 47(1), 29-98 (2011)

3. Böcherer, Siegfried, Das, Soumya: Characterization of Siegel cusp forms by the growth of their Fourier coefficients. Math. Ann. 359(1-2), 169-188 (2014)

4. Das, S.: On the natural densities of eigenvalues of a Siegel cusp form of degree 2. Int. J. Numb. Theory 9(01), 9-15 (2013)

5. Das, S.: Omega results for Fourier coefficients of half-integral weight and Siegel modular forms. In: Ramakrishnan, B., Heim, B., Sahu, B. (eds.) Modular Forms and Related Topics in Number Theory: Kozhikode, India, December 10-14. Springer, New York (2018)

6. Das, S., Sengupta, J.: An Omega-result for Saito-Kurokawa lifts. Proc. Am. Math. Soc. 142(3), 761764 (2014)

7. Das, S., Kohnen, W., Sengupta, J.: On a convolution series attached to a Siegel Hecke cusp form of degree 2. Ramanujan J. 33(3), 367-378 (2014)

8. Gun, S, Paul, B, Sengupta, J.: On Hecke eigenvalues of Siegel modular forms in the Maass space. In: Forum Mathematicum. Vol. 30. 3. De Gruyter. pp. 775-783 (2018)

9. Kim, H.H., Wakatsuki, S., Yamauchi, T.: An equidistribution theorem for holomorphic Siegel modular forms for GSp4 and its applications. J. Inst. Math. Jussieu (2018). https://doi.org/10.1017/ S147474801800004X

10. Kohnen, W.: Sign changes of Hecke eigenvalues of Siegel cusp forms of genus two. In: Proceedings of the American Mathematical Society, pp. 997-999 (2007)

11. Liu, J., Ye, Y.: Perron's formula and the prime number theorem for automorphic L-functions. Pure Appl. Math. Q. 3(2), 481-497 (2007)

12. Pitale, A., Saha, A., Schmidt, R.: Transfer of Siegel cusp Forms of Degree 2, vol. 232. American Mathematical Society, Providence (2014)

13. Ram Murty, M.: Oscillations of Fourier coefficients of modular forms. Math. Ann. 262(4), 431-446 (1983)

14. Rankin, R.A.: An $\Omega$ result for the coefficients of cusp forms. Math. Ann. 203(3), 239-250 (1973)

15. Saha, A.: Prime density results for Hecke eigenvalues of a Siegel cusp form. Int. J. Numb. Theory 7(04), 971-979 (2011)

16. Tsuzuki, M.: Spectral average of central values of automorphic L-functions for holomorphic cusp forms on $\mathrm{SO}_{0}(\mathrm{~m}, 2)$, II. (2019) arXiv: 1906.01172

17. Weissauer, R.: Endoscopy for GSp(4) and the Cohomology of Siegel Modular Threefolds. Springer, New York (2009)

Publisher's Note Springer Nature remains neutral with regard to jurisdictional claims in published maps and institutional affiliations. 